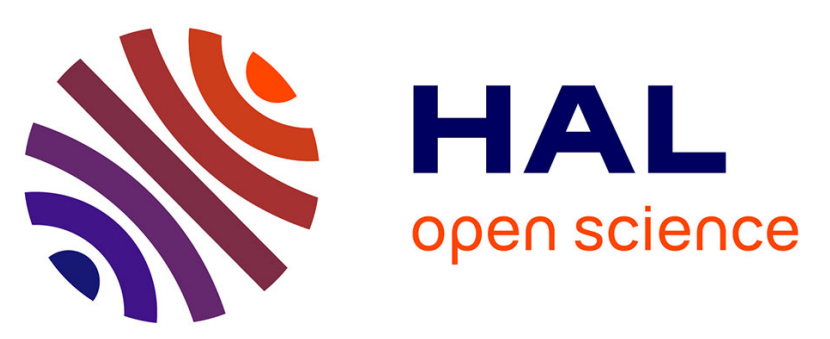

\title{
Binding modes of thrombin binding aptamers investigated by simulations and experiments
}

\author{
Ana Trapaidze, Aurélien Bancaud, Marie Brut
}

\section{To cite this version:}

Ana Trapaidze, Aurélien Bancaud, Marie Brut. Binding modes of thrombin binding aptamers investigated by simulations and experiments. Applied Physics Letters, 2015, 106 (4), pp.043702. 10.1063/1.4906594. hal-01137420

\section{HAL Id: hal-01137420 \\ https://hal.science/hal-01137420}

Submitted on 30 Mar 2015

HAL is a multi-disciplinary open access archive for the deposit and dissemination of scientific research documents, whether they are published or not. The documents may come from teaching and research institutions in France or abroad, or from public or private research centers.
L'archive ouverte pluridisciplinaire HAL, est destinée au dépôt et à la diffusion de documents scientifiques de niveau recherche, publiés ou non, émanant des établissements d'enseignement et de recherche français ou étrangers, des laboratoires publics ou privés. 


\title{
Binding modes of thrombin binding aptamers investigated by simulations and experiments
}

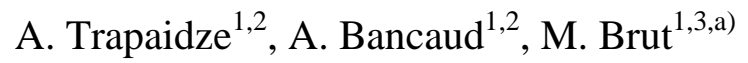 \\ ${ }^{1}$ CNRS, LAAS, 7 avenue du colonel Roche, F-31400 Toulouse, France \\ ${ }^{2}$ Université de Toulouse, LAAS, F-31400 Toulouse, France \\ ${ }^{3}$ Université de Toulouse, UPS, LAAS, F-31400 Toulouse, France
}

a) Author to whom correspondence should be addressed. Electronic mail: marie.brut@laas.fr.

\begin{abstract}
Thrombin binding aptamers HD1 and HD22 are the most studied aptamers, both for therapeutic and sensing purposes. Yet there is still no commercialized aptamer-based sensor device for thrombin detection, suggesting that the binding modes of these aptamers remain to be precisely described. Here we investigate thrombin-aptamer interactions with molecular dynamics simulations, and show that the different solved structures of HD1-thrombin complex are energetically similar and consequently possibly co-existing. Conversely HD22 folding is much more stable and its binding energy with thrombin is significantly largerthan that of HD1 complexes. These results are confronted to experiments, which consist in monitoring aggregation of aptamer-functionalized gold nanoparticles triggered by thrombin. HD1 alone, but not HD22, can trigger aggregation, meaning that this aptamer has multiple sites of interactions with thrombin. Furthermore, pre-incubation of HD22 with thrombin impedes HD1 aggregation, suggesting that HD1 and HD22 have competing affinities for the same binding site. Altogether this study shows that thecharacterization of aptamer-thrombin interactionsby structural and kinetic experiments joined to simulations is necessary for the development of biosensors.
\end{abstract}

Aptamers are single-stranded nucleic acids able to bind to a variety of targets with high specificity and affinity ${ }^{1-3}$. Their selection is achieved through the SELEX protocol, which consists in consecutivecycles of selection and amplification, starting from a large library of oligonucleotides $\left(10^{13}-10^{18} \text { sequences }\right)^{4}$. Due to their attractive properties in term of ease of synthesis, low-cost, chemical stability, and high affinity and specificity for a broad range of targets, aptamers have attracted much attention, particularly as potential therapeutic inhibitors and sensing elements for a future generation of biosensors ${ }^{5}$. Thrombin binding aptamers (TBA) HD1 and HD22 have been the most studied aptamers for biosensing, representing $20 \%$ of the 5000 papers published about the use of aptamers for analytical technologies ${ }^{6}$.Surprisingly there is little consensus on their binding properties in the literature. A rapid survey of Surface Plasmon Resonance (SPR) data shows that the reaction constant of HD1 and HD22 spans 2 
decades from $6 \mathrm{nM}^{7}$ to $170 \mathrm{nM}^{8}$ and from $2 \mathrm{nM}^{9}$ to $110 \mathrm{nM}^{8}$ for HD1 and HD22, respectively. Efforts to characterize the interaction of aptamers with thrombin are thus needed to improve their performances as sensing layers in a device, and definitively establish aptamerbased technologies.

HD1, the 15-mer DNA (5'-GGTTGGTGTGGTTGG-3') TBA that binds the fibrinogen site, also known as exosite I, was the first to be described in $1992^{10}$ and structurally characterized in $1993^{11}$. HD1 was shown to adopt a G-quadruplex structure with one TGT and two TT loops (see Fig. 1). The loops have been shown to bind thrombin in different ways, according to X-ray and NMR models respectively ${ }^{12}$, andthe determination of multiple crystal structures of the thrombin-HD1 complex has left a doubt on the existence of a unique complex vs.several co-existing states ${ }^{13}$. On the other hand, only one structure of thrombin-HD22 complex has been reported in the literature. This 29-mer (5'AGTCCGTGGTAGGGCAGGTTGGGGTGACT-3'), which presents a duplex-quadruplex folding, binds to the heparin site, known as exosite II, with a higher affinity ${ }^{14}$ than HD1.

In this study, we considered all TBA structures available in the Protein Data Bank (PDB), namely four HD1- and one HD22- thrombin structures (Fig. 1). Our goal was to compare their energy in their equilibrium configuration, as well as their binding energy to thrombin. We first focused on the two HD1 low-resolution structures $(2.80 \AA)$ solved in $1996^{12}$ with NMRbased and X-ray-based models (PDB code: 1HAO and 1HAP respectively). In 1HAO, G8 and T9 nucleic bases from the TGT loop are stacked on the G-quadruplex while T7 is rejected outside the loop. Interaction with thrombin is established through both TT loops. In 1HAP, the same configuration of the TGT loop can be observed but it is directly interacting with

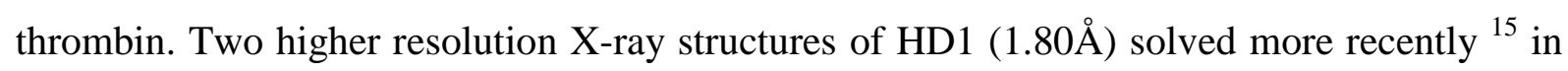
the presence of $\mathrm{Na}^{+}$and $\mathrm{K}^{+}$(PDB code: 4DIH and 4DII respectively) were also examined. $4 \mathrm{DIH}$ and 4DII chains follow the same orientation as 1HAO, and interaction with thrombin occurs through the TT loops. However the TGT loop undergoes a different configuration because T7 and G8 bases are now stacked on the G-quadruplex whereas T9 is rejected outside. The orientation of HD1 on thrombin is rotated by $180^{\circ}$ around the helix axis (Fig.1).The other meaningful difference comes from the cation position, which is inserted between the Gquartets anddrastically increases the inter-strand distance from $\sim 12 \AA$ in $1 \mathrm{HAO}$ and 1HAP to $\sim 21 \AA$ in 4DIH and 4DII. More details about interaction modes have already been described 
15,16. Concerning HD22, the X-ray structure recently solved by Krauss et al. with a $2.80 \AA$ resolution was used in the calculations (PDB code: $4 \mathrm{I} 7 \mathrm{Y})^{14}$.

All calculations were performed with Amber 10 suite of programs ${ }^{17}$ and the parm99/bsc0 force field ${ }^{18}$. As a first step, TBAs were submitted to molecular mechanics energy minimization. Simulations were performed in implicit solvent (Generalized Born approach ${ }^{19}$ ), and using the sander module of Amber, with 200000 steps of conjugate-gradient minimization without restraints and a cutoff of $12 \AA$. Resulting energies are presented in TableI. Despite changes in folding associated to the lower compaction of4DII and 4DIH vs. $1 \mathrm{HAO}$ and 1HAP, the four HD1 energies are similar, spanning -3.15 to $-3.02 \mathrm{kcal}^{\mathrm{mol}}{ }^{-1}$. It is therefore impossible to ensure that one HD1 conformation prevails over the other ones. Moreover, the energy of HD22 is $-5.98 \mathrm{kcal}^{\mathrm{mol}}{ }^{-1}$, i.e. twice as large as that of HD1. This result is expected due tothe existence of a longer G-quadruplex motif in comparison to HD1, plus four base-pair duplex. For this reason, HD22 is less likely to undergo conformational changes; however, there is no other PDB data to support this hypothesis.

As a second step, the binding energy of TBA with thrombin was estimated with molecular mechanics Poisson-Boltzmann surface area (MM-PBSA) method. This method combines molecular mechanics with continuum solvation models to exploit multiple snapshots extracted from molecular dynamics trajectories. It presents the advantage to be faster than other usual techniques ${ }^{20,21}$. Each aptamer-thrombin complex was solvated in a cubic box of water molecules extending at least $10 \AA$ outside the structures (TIP3P model ${ }^{22}$ ). We first equilibrated the solvated complexes with a short 2000 steps of conjugate-gradient minimization run, followed by $50 \mathrm{ps}$ of heating up to $300 \mathrm{~K}$ with $\mathrm{MD}, 50$ ps of density equilibration with weak restraints $\left(2 \mathrm{kcal} . \mathrm{mol}^{-1} . \AA^{-2}\right)$ and 500 ps of constant pressure equilibration with no restraint. All simulations were run using a cutoff of $8 \AA$. The equilibration and subsequent production were run with Langevin dynamics for temperature control, and the shake algorithm to impose constraints on bond lengths involving hydrogen atoms, allowing for a 2 fs time step. The production runs were performed during $10 \mathrm{~ns}$ of fully unrestrained MD and the collected results for each run, recorded every 5 ps, were used to estimate the aptamer binding energy. The resulting estimated binding energies are reported in Table I. The binding energies of $1 \mathrm{HAO}$ and 1HAP complexes is very similar (-47.03 and $-48.81 \mathrm{kcal}^{\mathrm{mol}}{ }^{-1}$, respectively), but approximately twice lower than that of HD22-thrombin complex $\left(-88.37 \mathrm{kcal}^{-\mathrm{mol}^{-1}}\right)$. However, in presence of cations and more specificially of $\mathrm{K}^{+}$, HD1-thrombin complexes gains in stability 
with binding energies of -60.29 and $-66.73 \mathrm{kcal}^{\mathrm{mol}} \mathrm{m}^{-1}$. Because monovalent cations are ubiquitously used in molecular biology assays, HD1 is unlikely to be found unbound to cations. Therefore4DIH and 4DII complexes are expected to prevail over 1HAO and 1HAP, and at least two states likely co-exist in experiments. At this step we wished to test this idea further with simple experiments.

We thus carried out experiments involvingTBA-capped gold nanoparticlesof $80 \mathrm{~nm}$ (AuNPs). We monitored the dynamics of aggregation, which occurs whenever thrombinmediates the formation of bonds between two functionalized AuNPs ${ }^{23,24}$. Aggregation kinetics was monitored by dynamic light scattering (DLS), which provides measurements of average hydrodynamic radius over time.In a typical experiment, TBA-AuNPs diluted in $100 \mu \mathrm{L}$ PBS buffer at the final concentration 0.1 OD at 540 nmwere covered with $0.85 \mathrm{nmol}$ ofthiol modified TBA(Eurogentec). The initial size of the NP was measured as the control assuring that there was no aggregation without thrombin (Sigma-Aldrich), which was then introduced ata concentration of $50 \mathrm{nM}$. Thrombin injection into HD1-AuNP and HD22-AuNP mixture led to rapid aggregation,as expected and shown in many other experiments(Fig. 2). Nextthe same experiment carried out with only one TBA on AuNPs showed rapid aggregation for HD1 and a slight onset in average diameter of $3 \pm 0.5 \mathrm{~nm}$ for HD22, which is consistent with the binding of thrombin to NPs.This result therefore shows the existence of multiple binding sites for HD1, in agreement with earlier reports on the weak association of HD1 to thrombin exosite $\mathrm{II}^{12,25}$.Further complementing experiments were conducted to clarify whether the second binding site for HD1 was indeed exosite II. We reasoned that the specificity of HD22 binding to thrombin exosite II could be used to block the access to this site by pre-incubation of thrombin withan excess of HD22for 20 min. This HD22-thrombin complex was theninjected into the HD1-AuNP solution, and aggregation did not take place. Rather the average diameterincreased by $15 \pm 2 \mathrm{~nm}$, in agreement with the formation of ternary complex between HD1AuNP, thrombin and HD22. Altogether our results indicate that HD1 has binding sites with thrombin towards exosite I and also at the vicinity of exosite II. We made a final round of experiments to estimate the binding energy of HD1 to these two sites. HD1-AuNP was incubated with excess of single-stranded DNA complementary to HD1 (ssHD1'), and this mixture was then injectedto a solution of HD22-AuNP and thrombin or only thrombin. Aggregation occurred normally in the latter case, but not in the former one (not shown).The successful aggregation suggests that HD1 binding to exosite 1 is favored energetically over HD1-HD1' pairing, whereas thrombin could not displace HD1' for binding HD1 to exosite II leading to a 
constant hydrodynamic radius. Given the energy of HD1-HD1' pairing of $-29.35 \mathrm{kcal}^{\mathrm{mol}} \mathrm{m}^{-1}$ (OligoAnalyzer IDT), our simulations are consistent with these estimates of HD1 binding to exosite 1 ,since all calculated binding energies range from -66.73 to $-47.03 \mathrm{kcal} \mathrm{mol}^{-1}$. Moreover the low energy of the secondary binding site of HD1 to exosite IIof less than $30 \mathrm{kcal}^{\mathrm{mol}}{ }^{-}$ ${ }^{1}$ likely accounts the absence of crystals of this complex.

In this paper, a theoretical study of all different thrombin-aptamer complexes available in the PDB is conducted to investigate the possible binding modes of HD1 and HD22 to thrombin. Simulations show that HD1 has multiple modes of interaction tothrombin exosite I, which possibly co-exist, and HD22 has higher affinity for exosite II. Experiments confirm that the binding landscape of HD1 to thrombin is not limited to exosite I, and suggest a secondary low-affinity interaction to the vicinity of exosite II. These observations call into question the relevance of HD1 for sensing thrombin in real conditions involving abundant proteins with residual non specific interactions. Consequentlythe selection of aptamers through the SELEX protocol is not sufficient to guarantee the successful design of biosensing systems. This result is consonant with our previous observation that the attachment of an aptamer to a surface was interfering with its folding and potentially with its interaction properties ${ }^{26}$. Altogether, we argue that structural data, molecular modeling, and kinetic experiments are required to collect the full benefits of aptamers in technological developments. 


\section{REFERENCES}

${ }^{1}$ K-M. Song, S. Lee and C. Ban. Sensors 12, 612 (2012).

${ }^{2}$ A. D. Ellington and J. W. Szostack, Nature 346, 818 (1990).

${ }^{3}$ C. Tuerk and L. Gold, Science 249, 505 (1990).

${ }^{4}$ J. L. Boots, K. Matylla-Kulinska, M. Zywicki, B. Zimmermann, and R. Schroeder. Genomic SELEX.

Handbook of RNA Biochemistry: Second, Completely Revised and Enlarged Edition, 1185-1206 (2014).

${ }^{5}$ M. Mascini, I. Palchetti and S. Tombelli. Angew. Chem. Int. Ed. 51, 1316 (2012).

${ }^{6}$ B. Deng, Y. Lin, C. Wang, F. Li, Z. Wang, H. Zhang, X.-F. Li and X. C. Le. Anal. Chim. Acta 837, 1 (2014).

${ }^{7}$ S. Davis. Biacore J.1, 29 (2014).

${ }^{8}$ P.-H. Lin, R.-H. Chen, C.-H. Lee, Y. Chang, C.-H. Chen and W.-Y. Chen.Colloids Surf. B Biointerfaces 88, 552 (2011).

${ }^{9}$ J. Müller, D. Freitag, G. Mayer and B. Pötzsch, B.J. Thromb. Haemost. JTH 6, 2105 (2008).

${ }^{10}$ L. C. Bock, L. C. Griffin, J. A. Latham, E. H. Vermaas, and J. J. Toole. Nature 356, 564 (1992).

${ }^{11}$ R. F. Macaya, P. Schultze, F. W. Smith, J. A. Roe, and J. Feignon, Proc. Natl. Acad. Sci. USA 90, 3745 (1993).

${ }^{12}$ K. Padmanabhan and A. Tulinsky, Acta Cryst. D 52, 272 (1996).

${ }^{13}$ I. Russo Krauss, A. Merlino, C. Giancola, A. Randazzo, L. Mazzarella and F. Sica. Nucleic Acids Res. 39, 7858 (2011).

${ }^{14}$ I. Russo Krauss, A. Pica, Merlino, A. Randazzo, E. Novellino, L. Mazzarella and F. Sica. Acta Cryst. D69, 2403 (2013).

${ }^{15}$ I. Russo Krauss, A. Merlino, A. Randazzo, E. Novellino, L. Mazzarella and F. Sica. Nucleic Acids Res. 40, 8119 (2012).

${ }^{16}$ A. Pica, I. Russo Krauss, A. Merlino, S. Nagatoishi, N. Sugimoto and F. Sica. FEBS Journal 280, 6581 (2013).

${ }^{17}$ D.A. Case, T.A. Darden, T.E. Cheatham, C.L. Simmerling, J. Wang, R.E. Duke, R. Luo, R.C. Walker, W. Zhang, K.M. Merz, B. Wang, S. Hayik, A. Roitberg, G. Seabra, I. Kolossvary, K.F. Wong, F. Paesani, J. Vanicek and L. Jian. Eur. Phys. J. E 35, 75 (2012).

${ }^{18}$ A. Perez, I. Marchan, D. Svozil, J. Sponer, T.E. Cheatham, C.A. Laughton and M. Orozco. Biophys. J. 92, 3817 (2007).

${ }^{19}$ C.S. Rapp, and R.A. Friesner. Proteins 35, 173 (1999).

${ }^{20}$ P.A. Kollman, I. Massova, C. Reyes, B. Kuhn, S. Huo, L. Chong, M. Lee, T. Lee, Y. Duan, W. Wang, O. Donini, P. Cieplak, J. Srinivasan, D.A. Case and T.E. Cheatham. Acc. Chem. Res. 33, 889 (2000).

${ }^{21}$ G. Rastelli, A. Del Rio, G. Degliesposti and M. Sgobba. J. Comput. Chem. 31, 797 (2010). 
${ }^{22}$ W.L. Jorgensen, J. Chandrasekhar, J.D. Madura, R.W. Impey and M.L. Klein. J. Chem. Phys. 79, 926 (1983).

${ }^{23}$ V. Pavlov, Y. Xiao, B. Shlyahovsky and I. Willner. J.A.C.S. 126, 11768 (2004).

${ }^{24}$ C.-C. Huang, Y.-F. Huang, Z. Cao, W. Tan and H.-T. Chang. Anal. Chem. 77, 5735 (2005).

${ }^{25}$ B. Pagano, L. Martino, A. Randazzo and C. Giancola. Biophys. J. 94, 562 (2008).

${ }^{26}$ M. Brut, A. Trapaidze, A. Estève, A. Bancaud, D. Estève, G. Landa, M. Djafari Rouhani. Appl. Phys. Lett. 100, 163702 (2012).

\section{ACKNOWLEDGMENTS}

We thank the CALMIP Supercomputer Center for CPU resources.

\section{SUPPLEMENTARY MATERIAL}

See supplemental material at [URL will be inserted by AIP] for more information about the aggregation assays described in this letter. 
Figure 1
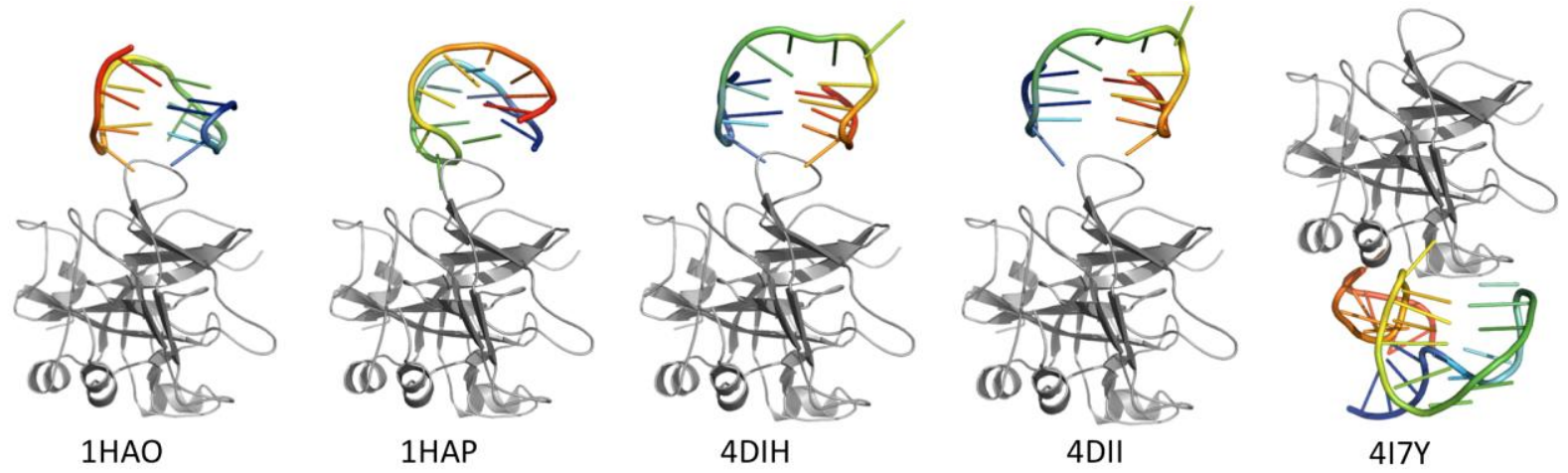

FIG. 1. Molecular structures of four HD1-thrombin complexes (1HAO, 1HAP, 4DIH, 4DII) and one HD22-thrombin complex (4I7Y) used in the calculations. Thrombin is colored in gray and aptamers in spectrum colors from blue to red to indicate the sequence orientation ( $5^{\prime}$ to $\left.3^{\prime}\right)$. 
Figure 2

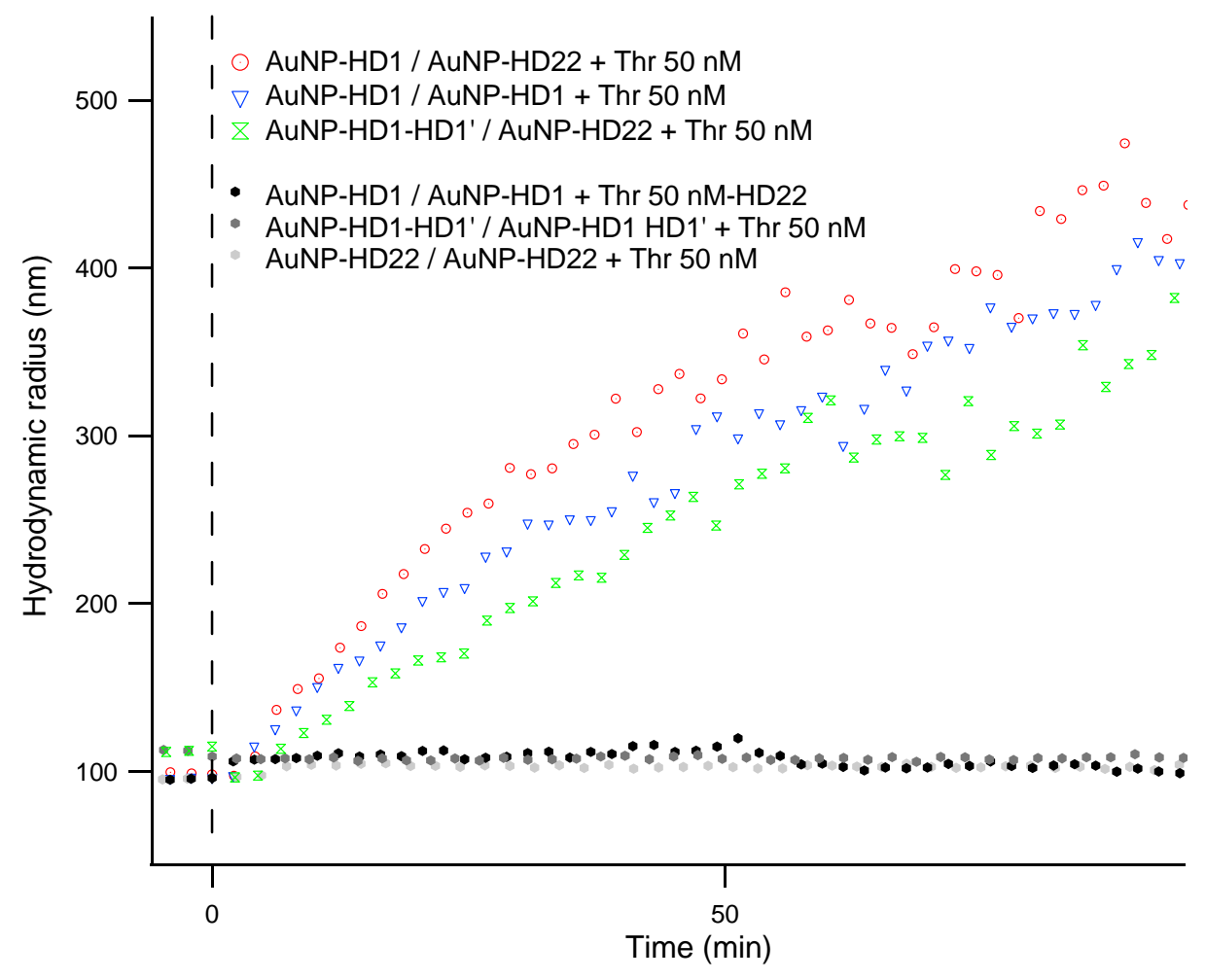

FIG. 2. Aggregation assay of $80 \mathrm{~nm}$ AuNPs coated with HD1 or HD22 aptamers. Initial time corresponds to the addition of $50 \mathrm{nM}$ thrombin in each vial. Aggregation is monitored by dynamic light scattering, which yields the average hydrodynamic radius over time. 
Table 1

\begin{tabular}{|l|c|c|c|c|c|}
\cline { 2 - 6 } \multicolumn{1}{c|}{} & $\begin{array}{c}\text { HD1 } \\
\text { (1HAO) }\end{array}$ & $\begin{array}{c}\text { HD1 } \\
(1 \mathrm{HAP})\end{array}$ & $\begin{array}{c}\text { HD1 } \\
(4 \mathrm{DIH})\end{array}$ & $\begin{array}{c}\text { HD1 } \\
\text { (4DII) }\end{array}$ & $\begin{array}{c}\text { HD22 } \\
\text { (4I7Y) }\end{array}$ \\
\hline Energy $\left(\mathrm{kcal} . \mathrm{mol}^{-1}\right)$ & -3.0241 & -3.0715 & -3.1594 & -3.1483 & -5.9810 \\
\hline$\Delta{\mathrm{G} \mathrm{Binding}\left(\mathrm{kcal}^{-} \mathrm{mol}^{-1}\right)}^{-47.0313}$ & -48.8083 & -60.2850 & -66.7253 & -88.3688 \\
\hline
\end{tabular}

Table I. Minimized energy of five aptamer structures extracted from the PDB, calculated with Amber 10 package, with the parm99/bsc0 force field and in implicit solvent. Binding energies of TBAs to thrombin are estimated with the MM-PBSA method. 